\title{
Constant-force cable coupler and activity monitor for small unrestrained animals
}

\author{
JAMES J. DESPOSITO, JEROME N. KING, and MICHAEL TERMAN \\ Northeastern University, Boston, Massachusetts 02115
}

\begin{abstract}
A mercury commutator with retracting cable coupler and activity monitor is described. A coiled spring maintains stable tension on a spool that takes up slack in a flexible cable attached to the electrode skull cap of a freely moving rat. This vertical movement-compensation assembly swivels on a low-friction horizontal bearing that follows the animal's circular movements. A sensitive measure of activity is achieved by using a photobeam to detect movement of the cable spool when the animal moves its head. The apparatus can be used with low-ceiling chambers and is suited to long-term continuous recording and stimulation experiments.
\end{abstract}

The use of chronic electrode implants for electrical recording and brain stimulation in unrestrained animals has become a routine laboratory procedure. A problem arises, however, when the animal must be maintained in the test environment continuously over long periods of time. Cable couplers developed for use in short-term experiments (Atrens \& Mendelson, 1970; Holley \& Powell, 1975; Sutton \& Miller, 1963) are not adequate. Neither these devices nor common techniques such as suspending a loop of cable by a light spring provide a means of vertical movement compensation that is sufficiently benign for long-term experimentation. One system provides both circular and vertical movement compensation with a counterweighted pulley arrange. ment (Berkley \& Kling, 1967). However, it requires the use of a nonstandard open-top chamber (from which an animal may escape), and can involve relatively high inertial loads at the animal's head because it does not consistently yield smooth cable extension and retraction when the animal is active. These factors may compromise the stability of chronic electrode implants.

In our laboratory, rats are connected continuously by skull-mounted cables to recording and stimulation apparatus for periods up to 1.5 years. The cable must remain out of the animal's reach and minimally restrict the animal's movement. To this end, the electrode cable is wrapped around a spool and anchored to the spool's rim by pulling it through a small hole in the rim. By connecting a coiled spring to the spool and its axle, the cable receives a retracting force that remains low and uniform regardless of the force exerted by the

Supported by PHS Grant MH27442. J. N. King had primary responsibility for the design and construction of the apparatus in fulfillment of undergraduate directed study requirements. We thank L. Thorington of the Duro-Test Corporation, for supplying Vita-Lites, and J. S. Terman, G. D. Ruben, and T. E. Phalen, Jr., for advice and technical assistance. Reprints may be obtained from J. Desposito, Department of Psychology, 234 United Building, Northeastern University, Boston, Massachusetts 02115 . A listing of the dimensions of each component is available upon request. rat to extract the cable, or the length of cable already extracted. The device is then mounted onto a platform so that it is free to rotate horizontally, thus yielding both vertical and circular movement compensation.

\section{CONSTANT-FORCE CABLE COUPLER}

The device described here meets the following requirements: (1) Rotation of the take-up spool in response to circular movements of the animal occurs with a minimum of torque; (2) vertical movement compensation occurs smoothly, with low constant force; and (3) the assembly can be used with standardsized, low-ceiling test chambers.

Figure 1 shows an exploded diagram of the device. The spring (h), $.087 \mathrm{in} .(.221 \mathrm{~mm})$ in thickness, is taken from the retraction mechanism of a self-retracting tape measure. The retracting force of the coiled spring is determined by the thickness of the spring steel and its

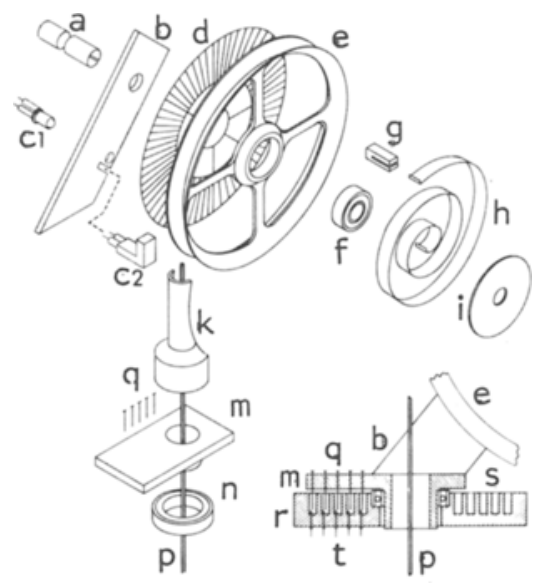

Figure 1. Exploded diagram of cable coupler and activity monitor: (a) stationary axle, (b) support arm, (c1) photodiode, (c2) photo detector, (d) photomask, (e) cable spool, (f) vertical bearing, (g) spring retainer, (h) spring, (i) spring guard, (k) cable guide, (m) platform, (n) horizontal bearing, (p) cable, (q) molybdenum wire, (r) base, (s) mercury wells, (t) molybdenum wire. 
relaxed curvature. Different thicknesses of spring steel can thus be accommodated by adjusting the curvature of the coil. The spring induces the cable spool (e; $12.7 \times 101.0 \mathrm{~mm}$ diam) to turn on the inner vertical bearing (f; Fafnir S3KDD-C3) and to retract the cable (p; Plastic Products) with a force of approximately $10 \mathrm{~g}$ and a maximum inertial load less than $40 \mathrm{~g}$. The bearing holds the spool in place by attaching to a stationary axle (a), which, in turn, fits into a support arm (b) cemented to a platform (m). A spring guard (i) insures that the spring opens and contracts without uncoiling. The spring is connected to the cable spool by a small retainer $(\mathrm{g})$ cemented to one spoke. Circular movement compensation is accomplished by the use of a low-friction horizontal bearing ( $\mathrm{n}$ : Barden A539) contained in the fixed base $\left(r ; 127 \mathrm{~mm}^{2}\right.$ ), inside of which the platform of the rotating assembly is tightly fit.

The electrical signals carried by the cable are transmitted by molybdenum (Mo) wires (q; City Chemical Corporation, New York) that protrude slightly above the rotating platform, as well as sufficiently below to be immersed in mercury $(\mathrm{Hg})$. The Mo wire is also fixed inside each $\mathrm{Hg}$ well ( $\mathrm{t}$ ) for connections to external apparatus. A thin hole is drilled into each well through the bottom of the Plexiglas base ( $\mathrm{r}$ ), and the wires are secured with a small amount of dental cement applied inside each well and on the bottom surface of the base. Mo wire has unusually high resistance to $\mathrm{Hg}$ corrosion and maintains excellent electrical contact (Berkley \& Kling, 1967).

\section{ACTIVITY MONITOR}

Figure 2 shows the mounted device with a cable connected to a rat's skull cap. Because of the direct cable connection, any motion of the animal's head is transferred to the spool, providing a simple means to monitor activity patterns. Movement of the spool is monitored by use of a photodiode and detector (Figure 1, c; GE LED56 and $1 / 2$ H13B1). The photobeam is interrupted by a photomask (d), with alternating opaque and transparent stripes, that is attached to the spool. Each interruption of the photobeam causes a relay closure, which enables recording of head movements in any direction as small as the width of the stripes ( $2 \mathrm{~mm}$, but adjustable for varying sensitivity).

Estimated cost of parts (bearings, springs, and Plexiglas) is less than $\$ 35$. The cable spools are machined from single pieces of .5 -in. Plexiglas, which requires considerable shop skill.

\section{ACTIVITY DATA}

Reliability of the activity monitor was tested by allowing an adult male ràt (LEW B/N, Microbiological

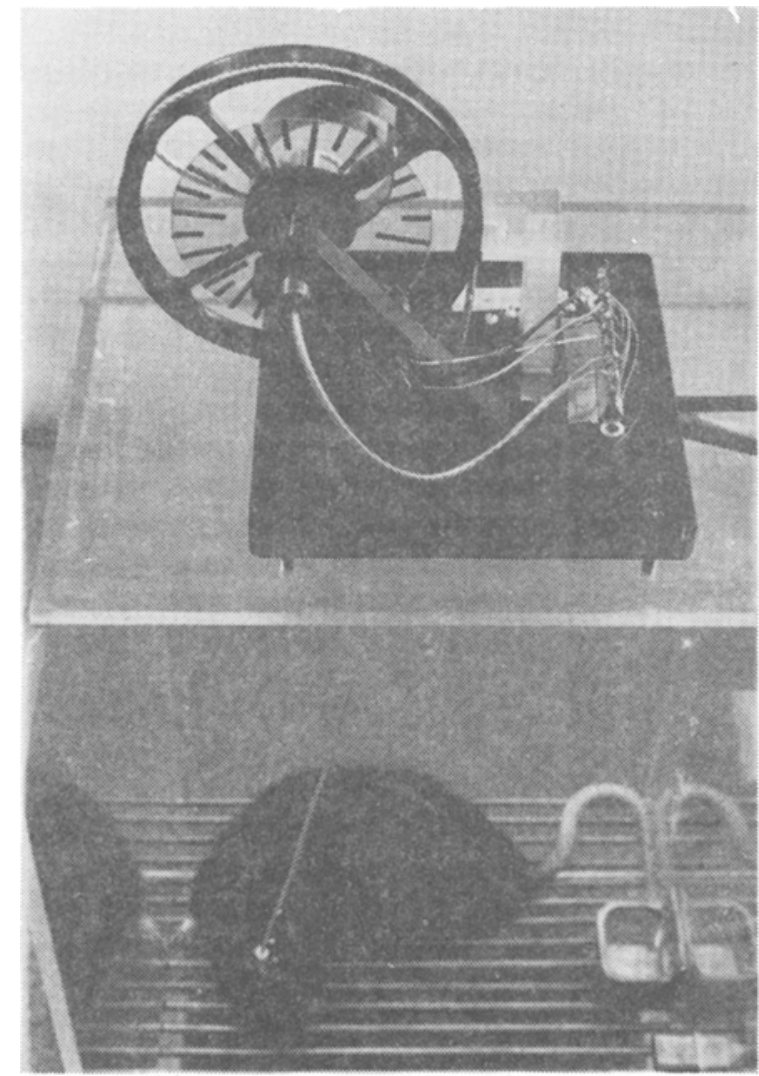

Figure 2. Photograph of device mounted on experimental chamber.

Associates) to live undisturbed in the test chamber with food (45-mg Noyes pellets) and water continuously available. A Vita-Lite fluorescent bulb provided a light-dark cycle of $12 \mathrm{~h}$ on and $12 \mathrm{~h}$ off.

Figure 3 shows a continuous $24-\mathrm{h}$ cumulative record of activity counts, comprising approximately 40,000 photobeam interruptions. In a typical hour of high activity, the recorder stepped approximately 5,000 times; in a typical hour of low activity, it stepped 500 times. While the relationship between topographical units of activity and the number of activity counts is not specified, the relative number of counts over time represents the relative level of general activity. All commonly observed spontaneous manifestations of the rat's activity, such as feeding, grooming, rearing, and even twitching during sleep, contribute to the record. When drinking occurs, the rat assumes a fixed head position with respect to the water spout. Thus, a drinking bout within an active segment results in a marked absence of activity counts for the duration of the bout. At light onset $(0600 \mathrm{~h})$, activity slows and remains at a relatively low level. Occasional extended bursts of activity during the daily light interval are almost always correlated with feeding bouts, as indicated by the diagonal slashes of the cumulative recorder pen. 

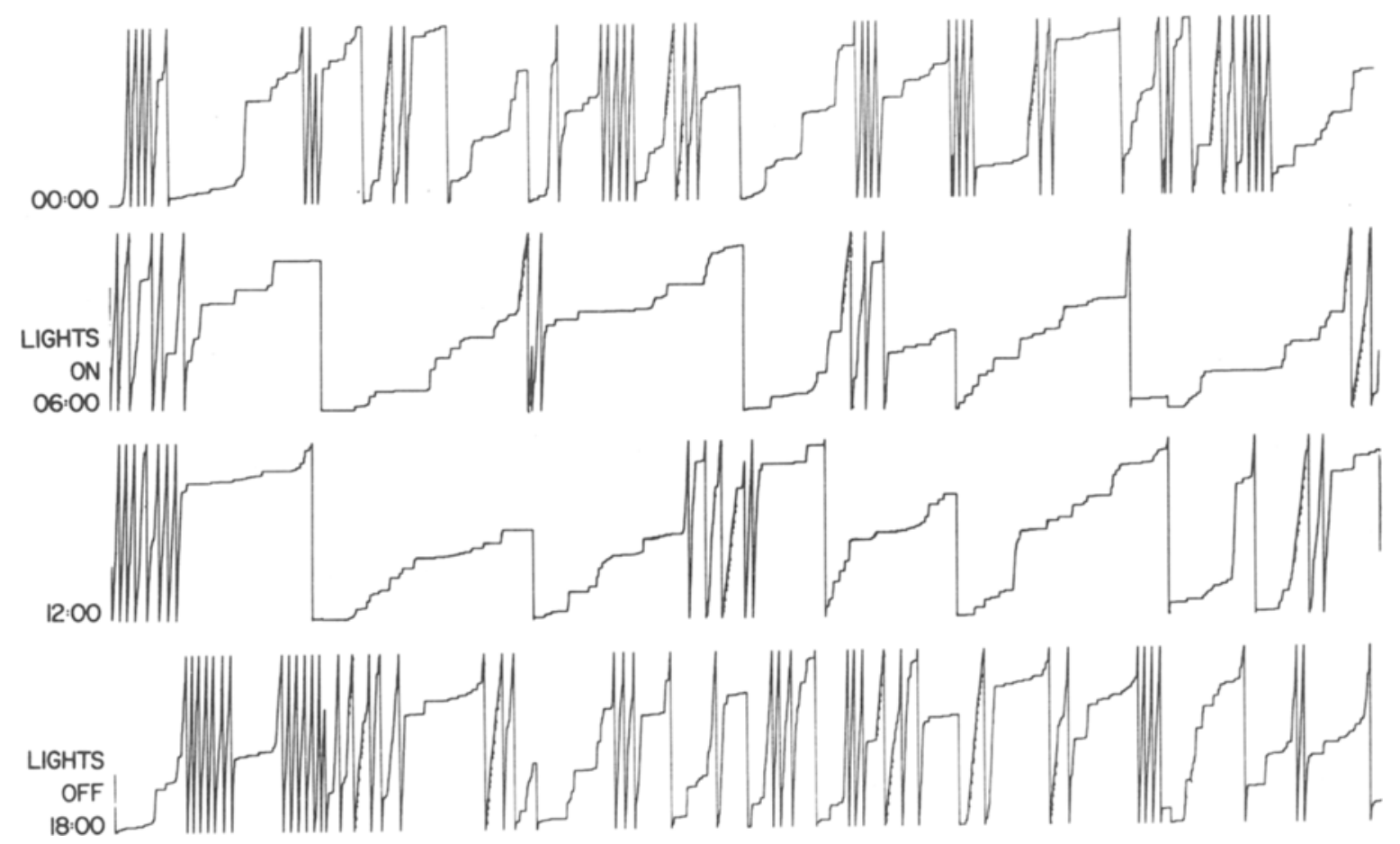

Figure 3. Cumulative record of activity counts. Food pellet deliveries are indicated by the small diagonal slashes. Each complete vertical excursion of the pen represents 500 activity counts. The pen resets after each excursion, as well as at the start of each hour. The light was turned on at $0600 \mathrm{~h}$, and off at $1800 \mathrm{~h}$.

The animal anticipates the time of light termination $(1800 \mathrm{~h})$ by a gradual acceleration of activity. Extended high levels of activity are maintained during the entire 12-h dark interval.

Figure 4 shows the results of a spectrum analysis performed on 18 consecutive days of hourly activity counts. The dominant period was $23.95 \mathrm{~h}$ (reflecting close entrainment to the 24-h light-dark cycle), with the daily peak at $2209 \mathrm{~h}$. The insert in Figure 4 presents the data in ranked quartile format. High-activity areas cluster in the early hours of dark, taper off gradually, remain low in the early hours of light (except for a feeding bout that often occurs at approximately $1000 \mathrm{~h}$ ), and increase in the $3-\mathrm{h}$ interval before light termination. The rapid rise in activity in the early hours of dark, followed by the gradual decrease, indicates that the daily waveform of activity output was asymmetrical, similar to a smoothed sawtooth. These data are similar in form to those collected by stabilimeter or ultrasonic devices. The simplicity of design, fixed calibration, and low cost of our device may favor its use, especially in experiments that require measurement of activity concurrent with brain stimulation or physiological recording.

\section{RECORDING APPLICATION}

The device described here is also effective for

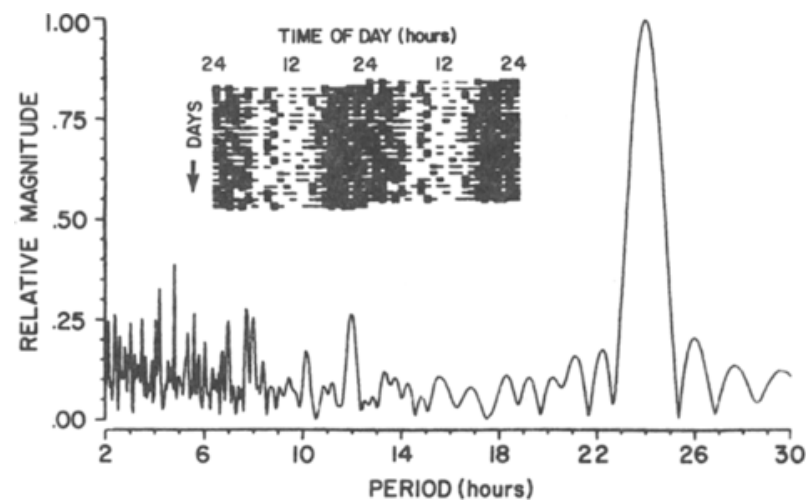

Figure 4. Spectrum analysis of a continuous 18-day sample of activity data. The insert shows the data in double-plotted ranked quartile format for which symbol size is proportional to the hourly distribution of activity counts.

recording deep-brain temperature. Using a chronically implanted thermistor in the preoptic hypothalamic area as the unstable leg in a Wheatstone bridge circuit, with a signal output ranging from 2 to $25 \mathrm{mV}$, stable recordings have been obtained continuously for more than 3 months. Figure 5 illustrates a $96-\mathrm{h}$ sample. This temperature record was obtained using a HewlettPackard $7100 \mathrm{~B}$ analog recorder. The full excursion of the record represents a temperature change of about $2^{\circ} \mathrm{C}\left(37.5^{\circ} \mathrm{C}-39.5^{\circ} \mathrm{C}\right)$. The record begins at the time of light termination $(1800 \mathrm{~h})$; during the ensuing 12 -h dark 


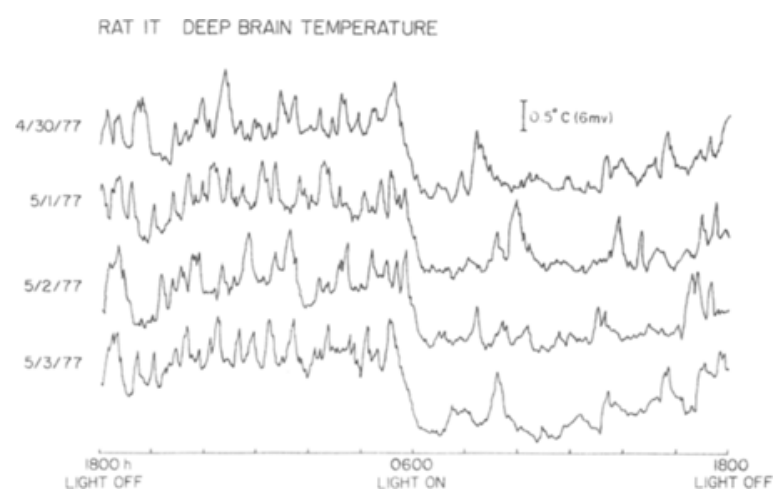

Figure 5. Tracing of a continuous $96-\mathrm{h}$ recording of deep-brain temperature in the rat under a light-dark cycle. The signal level ranges from approximately $2 \mathrm{mV}$ at the lowest point in the light to $25 \mathrm{mV}$ at the highest point in the dark.

interval, it is characterized by large and frequent changes in temperature level. Just before light onset $(0600 \mathrm{~h})$, deep-brain temperature drops to its daily low point and, except for an occasional increase at the time of a feeding bout, remains low for the next $8-10 \mathrm{~h}$. Several hours before the time of light termination, deep-brain temperature gradually begins to increase and, in anticipation of the dark interval, assumes its typical nocturnal pattern.
The 24 -h waveform is highly reliable. Similar results have been obtained using a subcortical thermocouple (Abrams \& Hammel, 1965) and intraperitoneal radio telemetry (de Castro \& Brower, 1977).

\section{REFERENCES}

Arrams, R., \& Hammel, H. T. Cyclic variations in hypothalamic temperature in unanesthetized rats. American Journal of Physiology, 1965, 208, 698-702.

Atrens, D. M., \& Mendelson, J. An inexpensive swivel for the delivery of brain stimulation to unrestrained animals. Physiology \& Behavior, 1970, 5, 707-708.

BerKLEY, M. A., \& KLING, J. W. A small animal cable coupler with vertical movement compensation. Physiology \& Behavior, 1967, 2, 315-316.

de Castro, J. M., \& Brower, E. Simple, reliable and inexpensive telemetry system for continuous monitoring of small animal core temperature. Physiology \& Behavior, 1977, 19, 331-333.

Holley, J. R., \& Powele, D. A. Mercury commutator arrangement for simultaneously stimulating or recording from two small animals in a social situation. Physiology \& Behavior, 1975, 15, 741-743.

Sutron, D., \& Mirler, J. M. Implanted electrodes: Cable coupler for elimination of movement artifact. Science, 1963, 140, 988-989.

(Received for publication March 20, 1978; accepted October 31,1978 .) 